\title{
COBERTURA DO EXAME CITOPATOLÓGICO DO COLO DO ÚTERO EM UM DISTRITO SANITÁRIO
}

\author{
COVERAGE OF THE CERVICAL CYTOPATHOLOGICAL \\ TEST IN A HEALTH DISTRICT
}

\section{COBERTURA DEL EXAMEN CITOPATOLÓGICO DEL CUELLO UTERINO EN UN DISTRITO SANITARIO}

\author{
Renata Barbosa Vilaça Marques de Carvalho ${ }^{1}$ \\ Mariluce Karla Bomfim de Souza²
}

\begin{abstract}
Como citar este artigo: Carvalho RBVM, Souza MKB. Cobertura do exame citopatológico do colo do útero em um Distrito Sanitário. Rev baiana enferm. 2021;35:e38463.

Objetivo: avaliar a cobertura do exame citopatológico do colo do útero. Método: estudo exploratório-descritivo com abordagem quantitativa, tendo como unidade de análise o Distrito Sanitário de Brotas da cidade de Salvador, Bahia. Foram estimadas as coberturas real e potencial do exame citopatológico do colo do útero realizado na Atenção Primária à Saúde, no ano de 2018. Para a estimativa das coberturas, o padrão foi estabelecido com base na recomendação do Ministério da Saúde. Os dados foram obtidos da Planilha de Monitoramento de Exame Citopatológico do Colo do Útero distrital disponibilizada pela Secretaria Municipal de Saúde. Resultados: foram encontradas baixas coberturas no período analisado, com cobertura real de 11,76\% e cobertura potencial de 46,11\%. Conclusão: a baixa cobertura do exame citopatológico do colo do útero pode estar relacionada com o pequeno crescimento da oferta do serviço devido à expansão insuficiente da Atenção Primária no município de Salvador.
\end{abstract}

Descritores: Cobertura de Serviços de Saúde. Papanicolau. Atenção Primária à Saúde. Saúde da Mulher.

Objective: to evaluate the coverage of the cervical cytopathological test. Method: exploratory-descriptive study with quantitative approach, whose unit of analysis was the Health District of Brotas of the city of Salvador, Babia. The study estimated the actual and potential coverage of the cervical cytopathological test performed in Primary Health Care in 2018. To estimate the coverage, the standard was established based on the recommendation of the Ministry of Health. The data were obtained from the Monitoring Worksheet of Cervical Cytopathological Test of the district provided by the Municipal Health Department. Results: there was low coverage in the analyzed period, with real coverage of $11.76 \%$ and potential coverage of $46.11 \%$. Conclusion: the low coverage of the cervical cytopathological test may be related to the small growth of the service offer due to the insufficient expansion of Primary Care in the city of Salvador.

Descriptors: Health Services Coverage. Papanicolaou Test. Primary Health Care. Women's Health.

Objetivo: evaluar la cobertura del examen citopatológico del cuello uterino. Método: estudio exploratorio-descriptivo con enfoque cuantitativo, teniendo como unidad de análisis el Distrito Sanitario de Brotas de la ciudad de Salvador, Babía. Se estimó la cobertura real y potencial del examen citopatológico del cuello uterino realizado en Atención Primaria de Salud en 2018. Para la estimación de la cobertura, la norma se estableció sobre la base

\footnotetext{
Nutricionista. Especialista em Saúde Coletiva. Universidade Federal da Bahia. Salvador. Bahia. Brasil. renatarussa90@hotmail.com. http://orcid.org/0000-0002-339I2394.

2 Enfermeira. Doutora em Saúde Pública. Professora Adjunta do Instituto de Saúde Coletiva da Universidade Federal da Bahia. Salvador. Bahia. Brasil. http://orcid. org/0000-0002-7895-4432.
} 
de la recomendación del Ministerio de Salud. Los datos fueron obtenidos de la Hoja de Seguimiento del Examen Citopatológico del Cuello Uterino del distrito proporcionada por la Secretaría de Salud Municipal. Resultados: se encontró baja cobertura en el periodo analizado, con una cobertura real del 11,76\% y una cobertura potencial del 46,11\%. Conclusión: la baja cobertura del examen citopatológico del cuello uterino puede estar relacionada con el pequeño crecimiento de la oferta de servicio debido a la insuficiente expansión de la Atención Primaria en la ciudad de Salvador.

Descriptores: Cobertura de los Servicios de Salud. Prueba de Papanicolaou. Atención Primaria de Salud. Salud de la Mujer.

\section{Introdução}

O câncer do colo do útero (CCU) é um importante problema de Saúde Pública, principalmente em países em desenvolvimento, ocupando o quarto tipo de câncer mais comum entre as mulheres, excluído pele não melanoma. Em 2018, foi responsável por aproximadamente 570 mil casos novos e 311 mil óbitos, com incidência de 13,1 casos por 100 mil mulheres e taxa de mortalidade de 6,9 casos por $100 \mathrm{mil}$ mulheres, no mundo ${ }^{(1)}$.

No Brasil, segundo dados do Instituto Nacional do Câncer (INCA), estimou-se cerca de 16 mil casos novos de CCU para cada ano do biênio 2018-2019, com risco estimado de 15,4 casos por 100 mil mulheres, ocupando o terceiro tipo de câncer mais frequente e a quarta causa de mortalidade (4,70 óbitos/100 mil). Quanto à distribuição por regiões, observa-se maior incidência e mortalidade na Região Norte (25,6 casos novos/100 mil), seguida pela Região Nordeste (20,5 casos novos/100 mil) e Centro-Oeste (18,3 casos novos/100 mil), evidenciando desigualdades regionais ${ }^{(2)}$.

Apesar do CCU apresentar alto potencial de prevenção, no Brasil, as taxas de incidência e mortalidade apresentam valores elevados quando comparadas às de países desenvolvidos com programas de detecção precoce organizados, como Austrália, Nova Zelândia e Ásia Ocidental $^{(2)}$. Mostra, dessa forma, que as medidas adotadas para o rastreamento da doença não estão sendo realizadas de forma sistemática e organizada, apresentando abrangência limitada e tendendo a negligenciar mulheres que mais se beneficiam com o exame ${ }^{(3-4)}$.
O exame citopatológico do colo do útero, popularmente conhecido como "preventivo" ou Papanicolau, é ainda a principal estratégia de rastreamento precoce do CCU devido à sua eficácia com até $85 \%$ de sensibilidade e especificidade, baixo custo, segurança e fácil execução, além de ser ofertado na Atenção Primária à Saúde (APS) ${ }^{(3,5)}$. Contudo, apesar da sua eficácia, a cobertura deste exame ainda é baixa para a população feminina brasileira.

A APS é a principal porta de entrada do Sistema Único de Saúde (SUS) e o centro de comunicação nas Redes de Atenção à Saúde (RAS), coordenando os fluxos e contrafluxos do sistema de Atenção à Saúde, incluindo o fluxo assistencial do CCU; devendo, portanto, desempenhar um papel central na garantia do cuidado ${ }^{(6-7)}$.

O Ministério da Saúde, por meio das diretrizes brasileiras para o rastreamento do câncer do colo do útero, preconiza a realização do exame de rastreamento em todas as mulheres que já iniciaram a vida sexual e que estejam na faixa etária entre 25 e 64 anos, com intervalo de três anos, após dois exames normais consecutivos realizados no intervalo de um ano ${ }^{(8)}$. Essa faixa etária está de acordo com as recomendações da Organização Mundial de Saúde (OMS).

A OMS preconiza uma cobertura de, no mínimo, $80 \%$ da população-alvo para obter o impacto epidemiológico esperado, isto é, redução, em média, de 60 a 90\% da incidência do $\mathrm{CCU}^{(9-11)}$. O Brasil estabeleceu como meta alcançar 85\% de cobertura do exame citopatológico do colo do útero até $2022^{(12)}$. 
De acordo com inquéritos populacionais, a cobertura do exame citopatológico variou de $82,8 \%$ a $83,8 \%$ nas capitais brasileiras, na faixa etária preconizada ${ }^{(13-14)}$, indicando, assim, coberturas acima do que é estabelecido pela OMS. Contudo, não obstante os avanços com relação ao aumento da cobertura, a redução da mortalidade por CCU ainda é um desafio a ser vencido no Brasil.

No caso de Salvador, capital da Bahia, das 120 Unidades Básicas de Saúde (UBS), 108 realizam a coleta de material para exame citopatológico, entretanto, os dados apontam baixa cobertura do exame citopatológico do colo do útero. Registra-se uma tendência de queda quanto ao número de exames citopatológicos realizados no período de 2010 a 2015, observando-se razões mais baixas nos anos de 2013 e $2014(0,17)$ e um discreto aumento em $2015(0,18)^{(15)}$.

No que tange aos dados sobre cobertura real e cobertura potencial dos serviços oferecidos na APS, constata-se escassez de estudos nos últimos dez anos com relação ao exame citopatológico do colo do útero, fazendo-se necessário conhecer se a oferta atende às necessidades dessa população. Assim, a fim de conhecer dados mais recentes sobre a cobertura do exame citopatológico e tendo em vista os territórios distritais que compõem a capital do estado da Bahia, questiona-se: Qual a cobertura real e potencial do exame citopatológico do colo do útero em um Distrito Sanitário (DS) de Salvador, Bahia, no ano de 2018?

Assim, constitui objetivo deste estudo: avaliar a cobertura do exame citopatológico do colo do útero em um DS da cidade de Salvador, Bahia, no ano de 2018.

\section{Método}

Trata-se de estudo exploratório, quantitativo, de caráter descritivo, tendo como unidade de análise o DS de Brotas, localizado no município de Salvador, Bahia, Brasil. A cidade de Salvador, capital da Bahia, está organizada em 12 DS. O DS estudado ocupa uma extensão territorial de $11,25 \mathrm{~km}^{2}$, com densidade demográfica de
19.950,49 habitantes $/ \mathrm{km}^{2}$, sendo considerado o terceiro distrito mais povoado, abrangendo 37 localidades $^{(15)}$. Possui no seu território 3 Unidades Básicas de Saúde e 3 Unidades de Saúde da Família (USF), com cobertura de APS de 37,61\% em 2018. Vale ressaltar que apesar do DS de Brotas possuir 6 unidades de saúde, somente 5 realizam o exame citopatológico do colo do útero (UBS 1 e UBS 2, USF 1, USF 2 e USF 3).

No ano de 2018, a população feminina estimada para o DS de Brotas foi de 118.089, correspondendo a $54,4 \%$ da população, sendo que: $26.068(22,07 \%)$ encontravam-se na faixa etária entre 10 e 24 anos; 69.401 (58,77\%) na faixa etária entre 25 e 64 anos; e 10.901 (9,23\%) na faixa etária maior ou igual a 65 anos de idade ${ }^{(16)}$.

Para avaliação da cobertura do exame citopatológico do colo do útero da rede própria do DS, utilizou-se como critério exames citopatológicos do colo do útero em mulheres entre 25 a 64 anos; como indicador, a cobertura do exame citopatológico do colo do útero em mulheres entre 25 a 64 anos; e como padrão, 0,33 exames/mulher/ano.

O padrão foi estabelecido com base na recomendação do Ministério da Saúde que preconiza a realização do exame citopatológico do colo do útero em um terço da população feminina, por ano, na faixa etária entre 25 a 64 anos de idade, atingindo $80 \%$ da população-alvo ${ }^{(8)}$.

No que tange às fontes de informação, foram utilizados os dados sobre a população feminina do DS disponibilizados no site do Tabnet - Salvador, com base na população estimada e no censo 2010, realizado pelo Instituto Brasileiro de Geografia e Estatística (IBGE) ${ }^{(16)}$. Para a obtenção do quantitativo de exames realizados pelo DS foram utilizados dados da Planilha de Monitoramento de Exame Citopatológico do Colo do Útero distrital, disponibilizados pela Secretaria Municipal de Saúde (SMS) de Salvador. Quanto à capacidade instalada de recursos humanos no DS para a realização do exame citopatológico do colo do útero, em 2018, foram utilizados os dados disponibilizados pelo Cadastro Nacional de Estabelecimentos de Saúde (CNES) para a identificação dos profissionais e 
a carga horária. Além disso, foram consultados os turnos semanais disponíveis nas unidades de saúde para a realização do procedimento.

Para o cálculo da cobertura real (CR) e potencial (CP) foram utilizadas as seguintes fórmulas:

$\mathrm{CR}=\mathrm{n}^{\mathrm{O}}$. procedimentos realizados em determinado ano $\mathrm{x} 100$

$\overline{\text { (População-alvo x padrão de concentração }}$ do procedimento por ano)
$\mathrm{CP}=\mathrm{n}^{\mathrm{o}}$ prof. $\mathrm{x}$ carga horária semanal $\mathrm{x} 4$ semanas $\mathrm{x}$ 11 meses $\mathrm{x}$ procedimentos-hora $\mathrm{x} 100$ (População-alvo x padrão de concentração do procedimento por ano)

Foi considerada como carga horária semanal, a carga horária semanal disponibilizada para a realização do procedimento, como apresentado na Tabela 1 a seguir:

Tabela 1 - Número de profissionais, carga horária semanal disponibilizada para a realização do exame citopatológico do colo do útero, por unidade de saúde, do Distrito Sanitário de Brotas. Salvador, Bahia, Brasil - 2018

\begin{tabular}{l|c|c|c}
\hline Unidade de Saúde & $\begin{array}{c}\text { No de } \\
\text { Profissionais }\end{array}$ & $\begin{array}{c}\text { Carga Horária } \\
\text { Semanal }\end{array}$ & $\begin{array}{c}\text { Carga Horária Semanal Disponibilizada } \\
\text { para a Realização do Procedimento }\end{array}$ \\
\hline Unidade Básica de & 1 & 40 & $4 \mathrm{~h}$ \\
Saúde 1 & 1 & 20 & $4 \mathrm{~h}$ \\
Unidade Básica de & 1 & 30 & $20 \mathrm{~h}$ \\
Saúde 2 & 1 & 40 & $16 \mathrm{~h}$ \\
Unidade de Saúde & 2 & 40 & $4 \mathrm{~h}$ \\
da Família 1 & & 40 & $4 \mathrm{~h}$ \\
Unidade de Saúde & 6 & & $4 \mathrm{~h}$ \\
de Família 2 & & 40 & \\
Unidade de Saúde & 5 & & \\
de Família 3 & &
\end{tabular}

Fonte: Elaboração própria.

Com relação ao número de procedimentos por hora, considerou-se o número de consultas de enfermagem por turno, estabelecido no Manual de Atenção Básica do Município de Salvador do ano de 2018, visto que grande parte dos exames citopatológicos do colo do útero são realizados por enfermeiras. Assim, o número de dez consultas de enfermagem por turno foi dividido por 4 h que corresponde a um turno, resultando em 2,5 procedimentos por hora.

Após a coleta foram utilizadas as frequências absolutas e relativas para as análises descritivas das variáveis categóricas. Os dados foram tabulados e analisados em planilhas do Microsoft Excel 2010 e estão apresentados em porcentagem.

Sobre os aspectos éticos, o projeto do estudo foi apreciado pela SMS de Salvador, que emitiu
Carta de Anuência para a sua realização. Em seguida, foi submetido ao Comitê de Ética em Pesquisa (CEP) do Instituto de Saúde Coletiva (ISC) e obteve aprovação segundo Parecer no 3.371 .664 e Certificado de Apresentação para Apreciação Ética (CAAE) no 14402319.0.0000.5030.

\section{Resultados}

No ano de 2018, foram realizados 2.693 exames do citopatológico do colo do útero pela rede própria do DS de Brotas. Dentre as unidades que ofertam este exame, a maioria foi realizado em unidades de saúde sem Estratégia Saúde da Família (ESF), correspondendo a 62,75\%, e alcançaram, em sua maior parte $(79,80 \%)$, mulheres na faixa etária entre 25 e 64 anos, população-alvo do rastreamento do CCU (Tabela 2). 
Tabela 2 - Frequência de exame citopatológico, por faixa etária, realizado pela rede própria do Distrito Sanitário de Brotas. Salvador, Bahia, Brasil - 2018. (N=2693)

\begin{tabular}{|c|c|c|c|c|c|c|c|c|}
\hline \multirow{3}{*}{ Unidade de Saúde } & \multirow{3}{*}{$\mathbf{n}$} & \multirow{3}{*}{$\%$} & \multicolumn{6}{|c|}{ Faixa etária } \\
\hline & & & \multicolumn{2}{|c|}{ 10-24 anos } & \multicolumn{2}{|c|}{ 25-64 anos } & \multicolumn{2}{|c|}{$\geq 65$ anos } \\
\hline & & & $\mathbf{n}$ & $\%$ & $\mathbf{n}$ & $\%$ & $\mathbf{n}$ & $\%$ \\
\hline Unidade Básica de Saúde 1 & 575 & 21,35 & 99 & 17,22 & 458 & 79,65 & 18 & 3,13 \\
\hline Unidade Básica de Saúde 2 & 1115 & 41,40 & 141 & 12,65 & 895 & 80,27 & 79 & 7,08 \\
\hline Unidade de Saúde de Família 1 & 197 & 7,32 & 35 & 17,77 & 158 & 80,20 & 4 & 2,03 \\
\hline Unidade de Saúde de Família 2 & 582 & 21,61 & 99 & 17,01 & 473 & 81,27 & 10 & 1,72 \\
\hline Unidade de Saúde de Família 3 & 224 & 8,32 & 50 & 22,32 & 165 & 73,66 & 9 & 4,02 \\
\hline Total & 2693 & 100,00 & 424 & 15,74 & 2149 & 79,80 & 120 & 4,46 \\
\hline
\end{tabular}

Fonte: Elaboração própria.

Na Tabela 3, que apresenta a frequência de exame citopatológico por faixa etária, observa-se que mais da metade dos exames (52,72\%) das mulheres que residem no DS de Brotas e, portanto, fazem parte da área de cobertura das unidades, foram realizados pelas USFs. Também se constata que não há diferença com relação à faixa etária quando comparado ao total de exames realizados pela rede própria do distrito.

Tabela 3 - Frequência de exame citopatológico, por faixa etária, em mulheres residentes no Distrito Sanitário de Brotas. Salvador, Bahia, Brasil - 2018. (N=1855)

\begin{tabular}{|c|c|c|c|c|c|c|c|c|}
\hline \multirow[t]{3}{*}{ Unidade de Saúde } & \multirow[t]{3}{*}{$\mathbf{n}$} & \multirow[t]{3}{*}{$\%$} & \multicolumn{6}{|c|}{ Faixa Etária } \\
\hline & & & \multicolumn{2}{|c|}{ 10-24 Anos } & \multicolumn{2}{|c|}{ 25-64 Anos } & \multicolumn{2}{|c|}{$\geq 65$ Anos } \\
\hline & & & $\mathbf{n}$ & $\%$ & $\mathbf{n}$ & $\%$ & $\mathbf{n}$ & $\%$ \\
\hline Unidade Básica de Saúde 1 & 550 & 29,65 & 97 & 17,64 & 435 & 79,09 & 18 & 3,27 \\
\hline Unidade Básica de Saúde 2 & 327 & 17,63 & 41 & 12,54 & 262 & 80,12 & 24 & 7,34 \\
\hline Unidade de Saúde de Família 1 & 181 & 9,76 & 30 & 16,57 & 147 & 81,22 & 4 & 2,21 \\
\hline Unidade de Saúde de Família 2 & 577 & 31,10 & 99 & 17,16 & 469 & 81,28 & 9 & 1,56 \\
\hline Unidade de Saúde de Família 3 & 220 & 11,86 & 50 & 22,73 & 161 & 73,18 & 9 & 4,09 \\
\hline Total & 1855 & 100,00 & 317 & 17,09 & 1474 & 79,46 & 64 & 3,45 \\
\hline
\end{tabular}

Fonte: Elaboração própria.

Considerando o território de abrangência e o distrito de residência, $68,88 \%$ ou 1.855 exames foram realizados em mulheres residentes do DS de Brotas, de modo que o percentual restante de pouco mais de $30 \%$ de mulheres que realizaram o exame nas unidades do distrito estudado não reside neste distrito. Dentre os DS com o maior quantitativo de mulheres que realizaram o exame na rede própria, estão os distritos da Liberdade (11,70\%), Cabula/Beirú (7,20\%) e Centro Histórico $(4,16 \%)$, respectivamente (Tabela 4 ).

Tabela 4 - Frequência de exame citopatológico, por distrito de residência, realizado pela rede própria do Distrito Sanitário de Brotas. Salvador, Bahia, Brasil - 2018. (N=2693)

(continua)

\begin{tabular}{l|c|c|c|c|c|c}
\hline $\begin{array}{l}\text { Unidade de } \\
\text { saúde/Distritos } \\
\text { Sanitários }\end{array}$ & $\begin{array}{l}\text { UBS 1 } \\
\mathbf{n ~ ( \% )}\end{array}$ & $\begin{array}{c}\text { UBS 2 } \\
\mathbf{n}(\%)\end{array}$ & $\begin{array}{c}\text { USF 1 } \\
\mathbf{n}(\%)\end{array}$ & $\begin{array}{c}\text { USF 2 } \\
\mathbf{n ~ ( \% )}\end{array}$ & $\begin{array}{c}\text { USF 3 } \\
\mathbf{n}(\mathbf{\%})\end{array}$ & $\begin{array}{c}\text { Total } \\
\mathbf{n}(\%)\end{array}$ \\
\hline Brotas & $550(95,65)$ & $327(29,33)$ & $181(91,88)$ & $220(98,21)$ & $577(99,14)$ & $1855(68,88)$ \\
$\begin{array}{l}\text { Barra/Rio } \\
\text { Vermelho }\end{array}$ & $2(0,35)$ & $20(1,79)$ & $4(2,03)$ & - & $1(0,17)$ & $27(1,00)$ \\
Boca do Rio & $3(0,52)$ & $4(0,36)$ & - & - & - & $7(0,26)$ \\
Cabula/Beirú & $5(0,87)$ & $183(16,41)$ & $4(2,03)$ & $2(0,89)$ & - & $194(7,20)$
\end{tabular}


Tabela 4 - Frequência de exame citopatológico, por distrito de residência, realizado pela rede própria do Distrito Sanitário de Brotas. Salvador, Bahia, Brasil - 2018. (N=2693)

(conclusão)

\begin{tabular}{lccc|c|c|c}
\hline $\begin{array}{l}\text { Unidade de } \\
\text { saúde/Distritos } \\
\text { Sanitários }\end{array}$ & $\begin{array}{l}\text { UBS 1 } \\
\mathbf{n}(\%)\end{array}$ & $\begin{array}{c}\text { UBS 2 } \\
\mathbf{n}(\%)\end{array}$ & $\begin{array}{c}\text { USF 1 } \\
\mathbf{n}(\%)\end{array}$ & $\begin{array}{c}\text { USF 2 } \\
\mathbf{n ~ ( \% )}\end{array}$ & $\begin{array}{c}\text { USF 3 } \\
\mathbf{n}(\%)\end{array}$ & $\begin{array}{c}\text { Total } \\
\mathbf{n}(\%)\end{array}$ \\
\hline Cajazeiras & $2(0,35)$ & $19(1,70)$ & $1(0,51)$ & - & - & $22(0,82)$ \\
Centro Histórico & - & $108(9,69)$ & - & $1(0,45)$ & $3(0,52)$ & $112(4,16)$ \\
Itapagipe & $3(0,52)$ & $11(0,99)$ & - & - & - & $14(0,52)$ \\
Itapuã & - & $15(1,35)$ & $4(2,03)$ & - & - & $19(0,71)$ \\
Liberdade & $6(1,04)$ & $306(27,44)$ & $1(0,51)$ & $1(0,45)$ & $1(0,17)$ & $315(11,70)$ \\
Pau Da Lima & - & $28(2,51)$ & - & - & - & $28(1,04)$ \\
São Caetano/ & - & $67(6,01)$ & $1(0,51 \%)$ & - & - & $68(2,53)$ \\
$\begin{array}{l}\text { Valéria } \\
\text { Subúrbio }\end{array}$ & $4(0,70)$ & $23(2,06)$ & - & - & - & $27(1,0)$ \\
$\begin{array}{l}\text { Ferroviário } \\
\text { Outros Municípios* }\end{array}$ & - & $4(0,36)$ & $1(0,51)$ & - & - & $5(0,19)$ \\
Total & $575(100)$ & $1115(100)$ & $197(100)$ & $224(100)$ & $582(100)$ & $2693(100)$ \\
\hline
\end{tabular}

Fonte: Elaboração própria.

Nota: Sinal convencional utilizado:

- Dado numérico igual a zero não resultante de arredondamento.

* Simões Filho, Lauro de Freitas e Santo Amaro.

UBS= Unidade Básica de Saúde; UFS= Unidade de Saúde de Família.

Vale ressaltar que o exame citopatológico do colo do útero ofertado pela rede própria do DS foi utilizado por mulheres residentes em todos os distritos sanitários de Salvador. Além disso, mulheres residentes em outros municípios do estado da Bahia também realizaram o procedimento em unidades do distrito estudado (Tabela 4).

Ainda na Tabela 4, é possível observar que a UBS 2, apesar de ser a unidade da rede própria que mais realiza o exame, é a unidade que menos atende às mulheres residentes no DS de Brotas (29,33\%).

No ano de 2018, considerando a população-alvo de 69.401 mulheres (faixa etária entre 25 e 64 anos), o número necessário de exames citopatológico do colo do útero foi de 22.902 exames, no entanto, o DS de Brotas realizou somente 2.693 exames, apresentando cobertura real muito baixa, de $11,76 \%$, não atingindo a sua cobertura potencial de $46,11 \%$. Tendo em vista essa diferença entre as coberturas potencial e real $(34,35 \%)$, observa-se que uma cobertura potencial maior não implicou em maior cobertura real ou melhor utilização da capacidade disponível.

Além disso, observa-se que, no ano de 2018, o DS de Brotas não conseguiu atingir a sua capacidade instalada de 10.560 exames por ano, realizando somente $25,50 \%$ da sua capacidade.

\section{Discussão}

Os achados apontam que, no ano de 2018, o DS de Brotas apresentou baixa cobertura do exame citopatológico do colo do útero, tendo em vista a oferta do procedimento pela rede própria. Destaca-se que se trata de um estudo com recorte distrital dentro de uma capital com baixa cobertura de APS.

A APS é responsável pela realização do exame citopatológico do colo do útero, utilizando como tecnologia o rastreamento ou screening, que se baseia na detecção precoce do CCU em mulheres assintomáticas. O Brasil tem como meta atingir, no mínimo, $80 \%$ a $85 \%$ da 
população-alvo, mulheres na faixa etária entre 25 e 64 anos de idade e que já iniciaram a vida sexual $^{(8,11-12)}$.

Embora o exame Papanicolau esteja disponível no SUS, principalmente na APS, ainda apresenta muitas barreiras de acesso e dificuldades quanto à continuidade do cuidado, contribuindo, assim, para o não cumprimento das metas de cobertura $^{(3,17-18)}$.

O DS de Brotas, no ano de 2018, obteve cobertura real muito baixa $(11,76 \%)$, porém atingiu a maioria das mulheres na faixa etária preconizada, 79,80\% da população-alvo. Dados do INCA apontam que 20\% a 25\% dos exames são realizados fora da faixa etária recomendada e quase a metade deles, com intervalo de 1 ano ou menos ${ }^{(8,19)}$.

Os resultados encontrados em estudos brasileiros utilizando dados da Pesquisa Nacional de Saúde (PNS) 2013 observaram cobertura do exame Papanicolau em 79,40\% das mulheres entre 25 e $64 \operatorname{anos}^{(3,5)}$. Resultado similar ao encontrado neste estudo.

Já em um município de grande porte populacional e com tecnologia disponível, localizado no estado de São Paulo, a cobertura do exame Papanicolau foi de aproximadamente 20\% ao ano, no período de 2008 a 2010. Além disso, a cobertura concentrou-se em mulheres mais jovens, não alcançando o grupo de mulheres com maior risco para o $\mathrm{CCU}^{(10)}$; o que não foi observado no presente estudo.

Observa-se na literatura a utilização de diversos métodos de avaliação de cobertura do exame citopatológico do colo do útero em diferentes faixas etárias e intervalos de rastreamento, dificultando, assim, a realização de comparações.

A menor cobertura foi observada na África do Sul, com aproximadamente $33 \%{ }^{(20)}$. Já a maior cobertura foi observada num estudo realizado na Finlândia, em que $87 \%$ das mulheres de 25 a 69 anos realizaram o exame Papanicolau nos últimos 5 anos, incluindo testes dentro e fora do programa de rastreamento organizado ${ }^{(21)}$.

Com relação à cobertura entre os estados brasileiros, verificou-se maior cobertura nos estados de Roraima (86,5\%), Santa Catarina $(84,5 \%)$ e Espírito Santo (84,2\%), e menores percentuais no Maranhão (67,7\%), Amapá (68,8\%) e Ceará (69,0\%). A Bahia apresentou percentual de $82,0 \%{ }^{(3)}$.

As Regiões Norte e Nordeste do Brasil apresentaram o maior percentual de mulheres entre 25 e 64 anos que nunca haviam realizado o teste de rastreamento do CCU e o menor percentual de exames realizados nos últimos 3 anos anteriores à pesquisa, demonstrando, assim, desigualdade na cobertura do exame citopatológico do colo do útero entre as regiões brasileiras ${ }^{(3)}$.

Trazendo para discussão a cobertura do exame citopatológico do colo do útero em áreas de cobertura de APS, ao analisar a cobertura e a adequação da periodicidade do exame Papanicolau em mulheres residentes em áreas de abrangência de UBS nas Regiões Sul e Nordeste do Brasil, no ano de 2005, foi verificada cobertura de $75,30 \%$, sem diferença significativa entre as Regiões Sul (74,30\%) e Nordeste (76,20\%). Além disso, observou-se que a periodicidade adequada da realização do exame foi de aproximadamente $71,00 \%$, sendo a maior parte na faixa etária preconizada. Apenas 38,20\% das mulheres relataram ter realizado o último exame na UBS da área de abrangência de sua moradia ${ }^{(22)}$. Esse dado corrobora o resultado aqui apresentado sobre a cobertura do procedimento para além da área, inclusive alcançando mulheres residentes em outros municípios.

Em Feira de Santana, município próximo a Salvador, foi realizado um estudo com mulheres entre 25 e 59 anos, residentes na zona urbana em áreas de abrangência da ESF, em 2010, que demonstrou alta cobertura de realização do Papanicolau (87,40\%), sendo que apenas $38,00 \%$ relataram ter realizado o exame na rede particular $^{(23)}$.

Inquérito realizado com usuárias de Unidades Básicas de Saúde participantes do Programa Nacional de Melhoria do Acesso e da Qualidade da Atenção Básica (PMAQ), em cinco regiões brasileiras, em 2012, demonstrou que a maioria $(79,4 \%)$ relatou ter feito o exame na UBS onde foram entrevistadas. Das mulheres que não realizaram na UBS de referência (14,0\%), 33,6\% 
relataram ter realizado em consultório particular, $31,3 \%$ em outra UBS, 10,6\% fizeram em hospital e $6,3 \%$ em outro local $^{(24)}$.

O presente estudo também apontou que a maioria dos exames foram realizados em mulheres residentes na sua área de abrangência. Nessa direção há que trazer particularidades do distrito sobre cobertura. Com destaque para a cobertura de exame citopatológico do colo do útero para as mulheres residentes no distrito sanitário a maior parte foi realizado em USF, o que é coerente com as diretrizes da estratégia, de modo que, a cobertura para as mulheres não residentes ficou a cargo das UBS que trabalham com demanda espontânea. Ressalta-se inclusive que as residentes de distritos vizinhos (Liberdade, Cabula-Beirú e Centro Histórico) acessaram o exame dentro do distrito estudado, tendo em vista que suas coberturas de APS também são baixas, o que evidencia o limite do município de Salvador quanto à APS.

Foram observadas no presente estudo discrepâncias entre a cobertura potencial e real, indicando, assim, possíveis problemas de implementação das ações. A cobertura potencial relaciona-se com a capacidade e possibilidade da oferta de serviços, medindo os recursos materiais e humanos para a realização das ações; já a cobertura real está relacionada com a utilização do serviço, correspondendo à proporção da população que efetivamente utilizou o serviço, ou seja, mede o trabalho realizado ${ }^{(25)}$.

Essa diferença entre a cobertura potencial e real pode estar relacionada a problemas de gestão de recursos disponíveis como, por exemplo, a subutilização da carga horária dos profissionais e ausência de planejamento e programação local em saúde.

Outros fatores também podem contribuir para menor cobertura do exame Papanicolau, como: falta de estrutura da unidade de saúde; baixa flexibilidade no agendamento; distância entre a UBS e a moradia; dificuldades de transporte; descrença da qualidade do exame realizado na unidade de abrangência; baixo nível socioeconômico; baixo grau de escolaridade; entendimento da importância da realização do exame; falta de orientação sobre a periodicidade do exame; constrangimento em ser examinada por profissionais do sexo masculino, dentre outros ${ }^{(3,10,17,24)}$.

O estudo apresentou como limitações a utilização de dados secundários, que podem interferir na análise, com viés de informação, sobretudo pela incompletude dos dados, subnotificação e registro incorreto, devendo os resultados serem interpretados com cautela. Além disso, não foram encontrados estudos que utilizassem o mesmo arcabouço metodológico para fins de comparações.

Vale ressaltar que foram identificados incompletude e/ou erro de digitação com relação a data de nascimento e bairro de residência em 2,9\% dos dados disponíveis na Planilha de Monitoramento de Exame Citopatológico do Colo do Útero distrital. Para correção dos dados foram utilizados o Sistema Integrado de Saúde ou Vida Mais, o site dos Correios e o Sistema de Informação de Câncer (SISCAN).

Outro ponto importante a ser destacado é que, não obstante o estudo de avaliação de cobertura ser um atributo básico e elementar na avaliação dos serviços de saúde e ser um componente indispensável para o processo de planejamento e gestão em saúde, apresenta limitações, pois não consegue aferir indicadores relacionados à organização da oferta e qualidade do cuidado, sendo necessário, portanto, ser complementado com outros estudos avaliativos que abordem outros atributos, como a acessibilidade, a efetividade e a qualidade técnico-científica.

A despeito das limitações apresentadas, este estudo traz contribuições importantes para o direcionamento de ações em saúde da mulher na APS do município de Salvador. Os resultados sobre a cobertura do exame citopatológico do colo do útero apresentados neste estudo podem ser úteis ao processo de planejamento, monitoramento e avaliação das ações em saúde da mulher, possibilitando a adequação e a organização do serviço.

\section{Conclusão}

Diante dos resultados apresentados, conclui-se que a baixa cobertura do exame citopatológico do colo do útero pode estar relacionada 
com o pequeno crescimento da oferta do serviço frente ao aumento populacional, com expansão insuficiente da APS no município de Salvador, gerando, assim, aumento da demanda assistencial.

Torna-se, então, necessário investimentos na APS para aumento da cobertura da ESF; capacitação de profissionais em planejamento e programação local em saúde; adequação dos processos de trabalho das unidades de saúde referente ao exame citopatológico do colo do útero; investimento tecnológico e melhoria no sistema de informação; adesão ao modelo de rastreamento organizado por meio da busca ativa dos casos mediante a sensibilização e mobilização das mulheres, associada ao monitoramento da realização do exame e do seguimento a fim de favorecer o aumento da cobertura, impactando na qualidade do programa de rastreamento do CCU.

Portanto, somente após a organização, qualificação e integração da APS com os demais níveis de atenção e implementação de ações da linha de cuidado de forma humanizada será possível diminuir a mortalidade por CCU no país.

\section{Colaborações:}

1 - concepção, projeto, análise e interpretação dos dados: Renata Barbosa Vilaça Marques de Carvalho e Mariluce Karla Bomfim de Souza;

2 - redação do artigo e revisão crítica relevante do conteúdo intelectual: Renata Barbosa Vilaça Marques de Carvalho e Mariluce Karla Bomfim de Souza;

3 - aprovação final da versão a ser publicada: Renata Barbosa Vilaça Marques de Carvalho e Mariluce Karla Bomfim de Souza.

\section{Referências}

1. World Health Organization. Globocan 2018 [Internet]. Geneva (CH): International Agency for Research on Cancer; 2018 [cited 2019 Mar 22]. Available from: https://gco.iarc.fr/

2. Instituto Nacional de Câncer José Alencar Gomes da Silva. Estimativa 2018: incidência de câncer no Brasil [Internet]. Rio de Janeiro (RJ); 2017 [cited 2019 Mar 20]. Available from: http://www.epi. uff.br/wp-content/uploads/2013/08/estimativaincidencia-de-cancer-no-brasil-2018.pdf

3. Barbosa IR. Regional and Socioeconomic Differences in the Coverage of the Papanicolau Test in Brazil: Data from the Brazilian Health Survey 2013. Rev Bras Ginecol Obstet. 2017;39(9):480-7. DOI: http://dx.doi.org/10.1055/ s-0037-1604481

4. Navarro C, Fonseca AJ, Sibajev A, Souza CIA, Araújo DS, Teles DAF, et al. Cervical cancer screening coverage in a high-incidence region. Rev Saúde Pública. 2015;49:17. DOI: https://doi. org/10.1590/S0034-8910.2015049005554

5. Oliveira MM, Andrade SSCA, Oliveira PPV, Silva GA, Silva MMA, Malta DC. Cobertura de exame Papanicolaou em mulheres de 25 a 64 anos, segundo a Pesquisa Nacional de Saúde e o Sistema de vigilância de fatores de risco e proteção para doenças crônicas por inquérito telefônico, 2013. Rev Bras Epidemiol. 2018;21:e180014. DOI: https://doi.org/10.1590/1980-549720180014

6. Brasil. Ministério da Saúde. Portaria no 2.436, de 21 de setembro de 2017. Aprova a Política Nacional de Atenção Básica, estabelecendo a revisão de diretrizes para a organização da Atenção Básica, no âmbito do Sistema Único de Saúde (SUS) [Internet]. Brasília (DF); 2017 [cited 2019 Mar 1]. Available from: https://bvsms.saude. gov.br/bvs/saudelegis/gm/2017/prt2436_22_09_ 2017.html

7. Mendes EV. As redes de atenção à saúde. Brasília: OPAS; 2011.

8. Instituto Nacional de Câncer José Alencar Gomes da Silva. Coordenação de Prevenção e Vigilância. Diretrizes brasileiras para o rastreamento do câncer do colo do útero [Internet]. Rio de Janeiro (RJ); 2016 [cited 2019 Mar 1]. Available from: https://www.inca.gov.br/sites/ufu.sti.inca.local/ files//media/document//diretrizesparaorastreamentodo cancerdocolodoutero_2016_corrigido.pdf

9. Audi CAF, Santiago SM, Andrade MGG, Francisco PMSB. Exame de Papanicolaou em mulheres encarceradas. Rev bras epidemiol. 2016;19(3):675-8. DOI: https://doi. org/10.1590/1980-5497201600030017

10. Brito-Silva K, Bezerra AFB, Chaves LDP, Tanaka OY. Integralidade no cuidado ao câncer do colo do útero: avaliação do acesso. Rev Saúde Pública. 2014;48:240-8. DOI: http://dx.doi. org/10.1590/S0034-8910.2014048004852 
11. World Health Organization. National cancer control programmes: policies and managerial guidelines [Internet]. Geneva (CH); 2002 [cited 2019 Mar 13]. Available from: https://apps.who.int/iris/bitstream/ handle/10665/42494/9241545577.pdf;jsessionid $=$ C871A11DEE90EDD65984E26CFBFAF920? sequence $=1$

12. Brasil. Ministério da Saúde. Plano de ações estratégicas para o enfrentamento das doenças crônicas não transmissíveis (DCNT) no Brasil 2011-2022 [Internet]. Brasília (DF); 2011 [cited 2019 Mar 22]. Available from: https:// portaldeboaspraticas.iff.fiocruz.br/wp-content/ uploads/2020/09/plano_acoes_enfrent_dcnt_2011. pdf

13. Brasil. Ministério da Saúde. Vigitel Brasil 2017. Vigilância de fatores de risco e proteção para doenças crônicas por inquérito telefônico: estimativas sobre frequência e distribuição sociodemográfica de fatores de risco e proteção para doenças crônicas nas capitais dos 26 Estados brasileiros e no Distrito Federal em 2017 [Internet]. Brasília (DF); 2018 [cited 2019 Mar 22]. Available from: https://bvsms.saude.gov. br/bvs/publicacoes/vigitel_brasil_2017_vigilancia_ fatores_riscos.pdf

14. Instituto Brasileiro de Geografia e Estatística. Pesquisa Nacional de Saúde 2013 - Módulo de Saúde da Mulher [Internet]. Rio de Janeiro (RJ); 2014 [cited 2019 Mar 17] Available from: http:// tabnet.datasus.gov.br/cgi/tabcgi.exe?pns/pnsr.def

15. Salvador. Secretaria Municipal da Saúde do Salvador. Diretoria Estratégica de Planejamento e Gestão. Plano Municipal de Saúde do Salvador 2018-2021 [Internet]. Salvador; 2018 [cited 2019 Mar 17]. Available from: http://www.saude. salvador.ba.gov.br/secretaria/wp-content/ uploads/sites/2/2018/12/Plano-Municipal-deSa\%C3\%BAde-2018-2021-VOLUME-I_aprovadopelo-CMS-21.11.pdf

16. Salvador. Secretaria Municipal de Saúde. Subcoordenação de Informação de Saúde. Tabnet-Salvador. [Internet]. Salvador; 2019 [cited 2019 Apr 8]. Available from: http://www.tabnet. saude.salvador.ba.gov.br/

17. Cardoso CL, Santos EF, França AMB, Cavalcante TCS, Lima KBM. Análise da cobertura de exames citopatológicos no estado de Alagoas. Semina cienc biol saúde [Internet]. 2014 [cited
2019 Mar 1;2(2):31-42. Available from: https:// periodicos.set.edu.br/fitsbiosaude/article/ view/1399/1040

18. Nascimento GWC, Pereira CCA, Nascimento DIC, Lourenço GC, Machado CJ. Cobertura do exame citopatológico do colo do útero no Estado de Minas Gerais, Brasil, no período entre 2000-2010: um estudo a partir dos dados do Sistema de Informação do câncer do Colo do Útero (SISCOLO). Cad saúde colet. 2015;23(3):253-60. DOI: http:// dx.doi.org/10.1590/1414-462X201500030059

19. Instituto Nacional de Câncer José Alencar Gomes da Silva. Plano de ação para redução da incidência e mortalidade por câncer do colo do útero: sumário executivo [Internet]. Rio de Janeiro (RJ); 2010 [cited 2019 Mar 1]. Available from: http://bvsms.saude.gov.br/bvs/publicacoes/ plano_acao_reducao_cancer_colo.pdf

20. Yunus NA, Yusoff HM, Draman N. Non-Adherence to recommended Pap smear screening guidelines and its associated factors among women attending health clinic in Malaysia. Malays Fam Physician [Internet]. 2018 [cited 2019 Mar 16;13(1):10-17. Available from: https://www.ncbi.nlm.nih.gov/ pmc/articles/PMC5962229/pdf/MFP-13-10.pdf

21. Salo H, Nieminen P, Kilpi T, Auranem K, Leino T, Vänskä $S$, et al. Divergent coverage, frequency and costs of organised and opportunistic Pap testing in Finland. Int J Cancer. 2014; 135(1):204-13. DOI: https://doi.org/10.1002/ ijc. 28646

22. Correia MS, Silveira DS, Siqueira FV, Fachini LA, Piccini RX, Thumé E, et al. Cobertura e adequação do exame citopatológico do colo uterino em estados das regiões Sul e Nordeste do Brasil. Cad Saúde Pública. 2012;28(12):2257-66. DOI: https:// doi.org/10.1590/S0102-311X2012001400005

23. Andrade MS, Almeida MMG, Araújo TM, Santos KOB. Fatores associados à não adesão ao papanicolau entre mulheres atendidas pela Estratégia Saúde da Família em Feira de Santana, Bahia, 2010. Epidemiol Serv Saúde. 2014; 23(1):111-20. DOI: https://doi.org/10.5123/ S1679-49742014000100011

24. Barcelos MRB, Lima RCD, Tomasi E, Nunes BP, Duro SMS, Facchini LA. Quality of cervical cancer screening in Brazil: external assessment of the PMAQ. Rev Saúde Pública. 2017;51:67. DOI: https:// doi.org/10.1590/s1518-8787.2017051006802 
25. Vieira-da-Silva LM. Avaliação de políticas e Recebido: 17 de agosto de 2020 programas de saúde. Rio de Janeiro: Fiocruz; 2014.

Aprovado: 9 de fevereiro de 2021

Publicado: 13 de abril de 2021

A Revista Baiana de Enfermagem utiliza a Licença Creative Commons - Atribuição-NãoComercial 4.0 Internacional. https://creativecommons.org/licenses/by-nc/4.0/

Este artigo é de acesso aberto distribuído sob os termos da Licença Creative Commons (CC BY-NC). Esta licença permite que outros remixem, adaptem e criem a partir do seu trabalho para fins não comerciais. Embora os novos trabalhos tenham de lhe atribuir o devido crédito e não possam ser usados para fins comerciais, os usuários não têm de licenciar esses trabalhos derivados sob os mesmos termos. 